\title{
Survey of outpatient sputum cytology: influence of written instructions on sample quality and who benefits from investigation
}

Kenneth W T Tsang, Andrew M Bentley, Jonathan S Mann, John Belcher, Charles F A Pantin

\begin{abstract}
Objectives - To evaluate quality of outpatient sputum cytology and whether written instructions to patients improve sample quality and to identify variables that predict satisfactory samples.

Design - Prospective randomised study.

Setting - Outpatient department of a district general hospital.

Patients - 224 patients recruited over 18 months whenever their clinicians requested sputum cytology, randomised to receive oral or oral and written advice.
\end{abstract}

Interventions - Oral advice from nurse on producing a sputum sample (114 patients); oral advice plus written instructions (110).

Main measures - Percentages of satisfactory sputum samples and of patients who produced more than one satisfactory sample; clinical or radiological features identified from subsequent review of patients' notes and radiographs associated with satisfactory samples; final diagnosis of bronchial cancer.

Results - 588 sputum samples were requested and 477 received. Patients in the group receiving additional written instructions produced $75(34 \%)$ satisfactory samples and $43(39 \%)$ of them one or more sets of satisfactory samples. Corresponding figures for the group receiving only oral advice $(80(31 \%)$ and $46(40 \%)$ respectively) were not significantly different. Logistic regression showed that radiological evidence of collapse or consolidation $(p<0.01)$ and hilar mass $(p<0.05)$ and history of smoking $(p<0.05)$ were significant predictors of the production of satisfactory samples. Sputum cytology confirmed the diagnosis in only $9(17 \%)$ patients with bronchial carcinoma.

Conclusions - The quality of outpatients' sputum samples was poor and was not improved by written instructions. Sputum cytology should be limited to patients with probable bronchial cancer unsuitable for surgery.

Implications - Collection of samples and requests for sputum cytology should be reviewed in other hospitals.
Introduction

Sputum cytology in outpatients is widely requested in medical clinics to investigate suspected bronchial carcinoma. ${ }^{1-6}$ Previous studies reported positive results of such examination in $29.1-90 \%$ of patients with proved bronchial carcinoma, ${ }^{47}$ and only $8-24 \%$ of patients failed to provide satisfactory sputum for examination. ${ }^{1-3}$ Duguid and Huish suggested that written instructions might improve the quality of sputum samples. ${ }^{1}$ However, no formal study has been reported. Most studies investigating the value of sputum cytology have been carried out on supervised inpatients. Many sputum samples, however, are requested from outpatients, who produce samples unsupervised. Therefore we designed a prospective study to assess the value of sputum cytology in outpatients in a district general hospital; the quality of the samples; the effect of giving written instructions to patients on the number of satisfactory samples; and the possible variables predicting which patients give satisfactory samples.

\section{Methods}

All patients attending the general medical outpatient department of this hospital between March 1987 and November 1988 in whom sputum cytology was requested were enrolled into the study (figure). They were given

Request for sputum cytology
Oral advice from nurse
Preservative free sputum pot
Envelope given to patient
(contents randomised)
appointment
instructions to
produce sputum
Specimen arrived at laboratory
(delivery by hand or post)
Sample regarded as satisfactory if alveolar
macrophages present
Initial screen by cytotechnologist
All specimens containing malignant and
suspicious or atypical cells examined by
consultant pathologist

Study design 
standardised oral advice by one of three designated clinic nurses on how to produce a satisfactory sputum sample. All were supplied with sputum pots free of preservative, which were to be delivered directly by hand or by post to the laboratory. All patients received an envelope randomly allocated to contain either a reminder for them to keep their next clinic appointment or written instructions reinforcing the oral advice (box).

In the laboratory two smears were made of all sputum samples, which were then fixed and stained. ${ }^{8}$ The samples were initially examined by a trained cytoscreener. Satisfactory or negative samples were reported as such. All samples were reviewed by a senior technician and, if regarded as abnormal, were examined by a consultant pathologist. Only those sputum samples containing alveolar macrophages were regarded as of lower respiratory tract origin and hence satisfactory. ${ }^{9}$

To identify possible variables predicting which patients give satisfactory samples all patients' notes and chest radiographs were reviewed at least 12 months after sputum cytology by KWT, AMB, and CFAP, and the final diagnosis was established. Bronchial carcinoma was diagnosed on the basis of histological findings. In the absence of a tissue diagnosis appropriate chest radiological evidence and clinical features and course were accepted as diagnostic.

Statistical analysis - Logistic regression ${ }^{10}$ was used to identify significant factors in the production of satisfactory sputum samples, with a significance level of $5 \%$.

\section{Results}

Two hundred and twenty four patients were recruited into the study (147 male, 77 female; mean age 62 (range 17-92)). One hundred and fourteen received only oral advice on sputum production whereas 110 received additional written instructions. One hundred and eighty patients had been smokers, and 44

\section{Instructions to patients for producing sputum specimens}

1 Sputum needs to be obtained first thing in the morning

2 The mouth should be free of food, so produce your specimens before breakfast and before cleaning your teeth

3 To obtain a sputum specimen cough deeply to bring the sputum from the lungs and not from the throat or mouth

4 The sputum needs to go straight into the special container(s) that the nurse provided you with. Then the lid needs to be put on and tightened

5 Please make sure that you write your name, the date, and the time on the container(s)

6 When the above is completed please place the container(s) into the plastic bag supplied and either bring them to the central pathology laboratory as soon as possible or return them by earliest post in the stamped envelope provided

Thank you for your cooperation had never smoked. There were no significant differences between the two groups in age, sex, and history of smoking.

In total, 588 sputum samples were requested, and 477 were received. The mean number requested per patient was 2.6. In the group receiving only oral advice $80(31 \%)$ of received samples were satisfactory compared with $75(34 \%)$ in the group receiving written instructions, and $46(40 \%)$, and $43(39 \%)$ in these groups respectively produced one or more satisfactory sputum samples in the set requested.

Fifty four patients were found to have bronchial carcinoma on review of their notes and chest radiographs 12 months or more subsequently (22 in the group receiving written instructions, 32 in the other group). Bronchoscopy confirmed the diagnosis in $32(59 \%)$ patients, sputum cytology in nine $(17 \%)$, and chest radiolography with clinical course in $13(24 \%)$. Sixty one (35\%) samples received in patients with bronchial carcinoma were satisfactory. Five $(2 \%)$ samples from the group receiving only oral advice contained malignant cells and none from the group receiving written instructions; the figures for atypical or suspicious cells were three $(1 \%)$ and five $(2 \%)$ respectively. Four $(2 \%)$ patients and seven $(3 \%)$ patients produced at least one sample containing malignant and suspicious or atypical cells respectively. Even when atypical or suspicious cells were regarded as indicating underlying malignancy the sensitivity of sputum cytology for detecting bronchial carcinoma was only $20 \%$. In two patients who subsequently underwent surgical resection the findings on sputum cytology had been negative. Both had had fibreoptic bronchoscopy to provide a histological diagnosis.

Logistic regression was performed on age; sex; smoking; written instructions; haemoptysis; cough; pleural effusion; weight loss; and chest radiographic changes of peripheral mass, hilar mass, and collapse or consolidation. The significant predictors of obtaining satisfactory sputum samples from patients were smoking $(p<0.05)$ and chest radiographic changes of collapse or consolidation $(p<0.01)$ and hilar mass $(\mathrm{p}<0.05)$.

\section{Discussion}

This study shows that most $(68 \%)$ of outpatient sputum samples submitted to one district general hospital's cytology department were of poor quality and inadequate for detecting bronchial carcinoma. The addition of written instructions to oral instructions alone failed to increase the total number of samples received or the number that were satisfactory. The detection rate of bronchial carcinoma is lower than previous reports of $29.1-90 \%,{ }^{7}$ even when atypical or suspicious cells were included as indicating underlying malignancy.

Many studies have shown the value of sputum cytology in detecting bronchial carcinoma. ${ }^{1-7}$ Most of them however, entailed retrospective analyses of patients with proved bronchial carcinoma and were carried out on 
a mixture of hospital inpatients and outpatients whereas this study was performed prospectively on outpatients.

The nine district general hospitals in the north of the West Midlands region were contacted to ascertain how outpatient sputum samples for cytology were collected, returned to the laboratory, and processed. Only one hospital added fixative to the sputum containers. Two utilised the postal service for return of the samples; otherwise samples were returned directly by patients. Eight hospitals employed cytoscreeners for screening of samples. All cytology departments used Papanicolau's staining method ${ }^{8}$ and accepted a sample as satisfactory if alveolar macrophages were present.

In two prospective studies of outpatients $92.5 \%$ and $70 \%$ respectively of sputum samples received for cytological examination were satisfactory. ${ }^{11} 12$ One study used a three day pooled sample for analysis, ${ }^{11}$ and both used sputum pots containing fixative. Fixative was not used in our study, and this may partly explain the poor quality of samples we received. Many of the samples were delivered to the laboratory by post, and excessive delay may have resulted in autolysis of cells. ${ }^{13}$ However, this may not fully explain the discrepancy in our results and those of previous studies. The low number of satisfactory samples we obtained may be partly explained by inappropriate requests in patients with little or no sputum, whose samples may have originated largely from the upper respiratory tract. Discriminatory statistical analysis showed that patients who smoked or had evidence of consolidation or collapse on the chest radiograph, or both, were significantly more likely to produce satisfactory sputum samples than those who did not - for example, non-smokers, patients with clear chest radiographs, or those showing only pleural effusion.

Previous studies questioned the cost effectiveness of sputum cytology as most patients shown to have malignant cells in their sputum subsequently undergo bronchoscopy to assess their suitability for surgery. ${ }^{14}{ }^{15}$ In this study $76 \%$ of all patients with a final diagnosis of bronchial carcinoma underwent broncho-scopy, including all four with positive results on sputum cytology and two of the seven patients with suspicious or atypical cells in their sputum. The cost of materials used in examining a sputum sample is low (20p), but the test is time consuming, a cytoscreener taking a minimum of 15 minutes to examine one sample.
The value of a negative result is less measurable, but the high rate of false negatives associated with sputum cytology is such that a negative result cannot be justifiably used to reassure the doctor or the patient. ${ }^{7}$ It may be for the reassurance of a negative result, however, that the inappropriate request is often made.

The results of this study support the suggestion that requests for sputum cytology should be reserved for patients in whom the diagnosis of lung cancer is probable but who would not be considered candidates for surgery. ${ }^{16}$ Sputum cytology is widely requested for outpatients in hospitals throughout England and Wales, often using methods identical to those in this study. ${ }^{16}$ Our results suggest that the methods of sample collection and appropriate use of the sputum cytology service in individual hospitals should be reviewed.

We thank Dr T French, Mr Kemlo Rogerson, and their colleagues in cytoscreening services for screening the samples and helping to trace the results; Mrs Jan Cohen for typing the manuscript; and the outpatient nursing staff for their help.

1 Duguid HLD, Huish DW. Clinical evaluation of cytodiagnosis in bronchial carcinoma. $B M \mathcal{F}$ 1963;ii:28791 .

2 Oswald NC, Hinson KFW, Canti G, Miller AB. The diagnosis of primary lung cancer with special reference to sputum cytology. Thorax 1971;26:623-31.

3 Clee MD, Sinclair DJM. Assessment of factors influencing the results of sputum cytology in bronchial carcinoma. Thorax 1981;36:143-6.

$4 \mathrm{Ng}$ ABP, Horak CG. Factors significant in the diagnostic accuracy of lung cytology in bronchial washing and sputum samples. II. Sputum samples. Acta Cytol sputum samples.

5 Hartveit F. Time and place for sputum cytology in the diagnosis of lung cancer. Thorax 1981;36:299-302.

6 Lazo BG, Feiner LL, Seriff NS. A study of routine cytologic screening of sputum for cancer in 800 men consecutively admitted to a tuberculosis service. Chest 1974;65:646-9.

7 Benbassat J, Regev A, Slater PE. Predictive value of sputum cytology. Thorax $1987 ; 42: 165-72$.

8 Papanicolau GN. New procedure for staining vaginal smears. Science 1942;95:438-9.

9 Courcol RJ, Damien JM, Ramon P, Voisin C, Martin GR. Presence of alveolar macrophages as a criterion for determining the suitability of sputum specimens for bacterial culture. Eur $f$ Clin Microbial Infect Dis 1984;3:122-5.

10 Snedecor GW, Cochran WG. Statistical methods. Iowa: Ames, 1967.

11 Fontana RS, Sanderson DR, Taylor WF, Woolner LB, Miller WE, Muhm JR, et al. Early lung cancer detection: results of the initial (prevalance) radiologic and cytologic screening in the Mayo Clinic study. Am Rev Resp Dis 1984;130:561-5.

12 Ahlbom G, Winslov J. Outpatient sputum cytology in the diagnosis of lung cancer. Scand $\mathcal{F}$ Resp Dis 1977; 58:227-30.

13 Philps FR. The identification of carcinoma cells in the sputum. Br f Cancer 1954;8:67-96.

14 Savage PJ, Donovan WN, Dellinger RP. Sputum cytology in the management of patients with lung cancer. South Med $\mathcal{f} 1984 ; 77: 840-2$.

15 Korompai FL, Hayward RH. Sputum cytology in the diagnosis and treatment of lung cancer. South Med $\mathcal{f}$ 1979;72:1121-2.

16 Oswald NC, Hinson KFW, Canti G, Husain OAN, Girling DJ, Tall $R$, et al. Survey of the sputum cytology service in England and Wales. Thorax 1975;30:489-96. 